\title{
Pravastatin therapy during preeclampsia prevents long-term adverse health effects in mice
}

\author{
Nicola Garrett, ${ }^{1}$ Joaquim Pombo, ${ }^{1}$ Michelle Umpierrez, ${ }^{1}$ James E. Clark, ${ }^{2}$ Mark Simmons, ${ }^{1}$ \\ and Guillermina Girardi ${ }^{1,3}$ \\ 'Pregnancy Laboratory, Department of Women and Children's Health, Rayne Institute, St Thomas' Hospital, King's \\ College London, London, United Kingdom. 'King's College London BHF Cardiovascular Centre, Rayne Institute, St Thomas' \\ Hospital, London, United Kingdom. ${ }^{3}$ Queen's Medical Research Institute, University of Edinburgh, Edinburgh, United \\ Kingdom.
}

Preeclampsia (PE), associates with long-term increased risk for cardiovascular disease in women, suggesting that PE is not an isolated disease of pregnancy. It is not known if increased risk for long-term diseases is due to PE-specific factors or to prepregnancy renal and cardiovascular risk factors. We used a mouse model in which a WT female with normal prepregnancy health develops $P E$ to investigate if preeclampsia causes long-term cardiovascular consequences after pregnancy for mothers and offspring. Mothers exhibited endothelial dysfunction and hypertension after $P E$ and had glomerular injury that not only persisted but deteriorated, leading to fibrosis. Left ventricular (LV) remodeling characterized by increased collagen deposition and MMP-9 expression and enlarged cardiomyocytes were also detected after PE. Increased LV internal wall thickness and mass, increased end diastolic and end systolic volumes, and increased stroke volume were observed after PE in the mothers. Placenta-derived bioactive factors that modulate vascular function, markers of metabolic disease, vasoconstrictor isoprostane-8, and proinflammatory mediators were increased in sera during and after a preeclamptic pregnancy in the mother. Offspring of PE mice developed endothelial dysfunction, hypertension, and signs of metabolic disease. Microglia activation was increased in the neonatal brains after PE, suggesting neurogenic hypertension in offspring. Prevention of placental insufficiency with pravastatin prevented PEassociated cardiovascular complications in both mothers and offspring. In conclusion, factors that develop during PE have long-term, cardiovascular effects in the mother and offspring independent of prepregnancy risk factors.

Conflict of interest: The authors have declared that no conflict of interest exists.

Submitted: January 25, 2018

Accepted: March 9, 2018

Published: April 19, 2018

Reference information: JCI Insight. 2018;3(8):e120147. https://doi.org/10.1172/jci. insight.120147.

\section{Introduction}

Cardiovascular disease (CVD) is the leading cause of death for women in the world. One in every 3 female deaths in the United States are caused by CVD (1). Although heart disease is sometimes thought of as a "man's disease," around the same number of women and men die each year of heart disease. While detection and treatment are important, effective strategies for identifying and preventing risk factors remains paramount, in particular, the identification of gender-specific risk factors. Women who are at high risk for hypertension and associated kidney and CVD need early detection and management to ameliorate this socioeconomic global burden.

Preeclampsia (PE) is a multisystem progressive disorder characterized by the new onset of hypertension and end-organ dysfunction with or without proteinuria in the last half of pregnancy, as described in the new guidelines of the American College of Obstetricians and Gynecologists. Ten million women develop $\mathrm{PE}$ each year (2). As PE generally resolves with delivery of the placenta, PE was considered a transient condition. However, long-term renal and cardiovascular risks have been associated with a history of maternal placental syndromes such as PE, suggesting that PE is more than an isolated disease of pregnancy (3). Increased risk of developing future chronic hypertension and CVD has been observed in women diagnosed with PE during pregnancy compared with normotensive women (4-6). While the American Heart Association guidelines in 2011 recognized $\mathrm{PE}$ as cardiovascular risk factor (7), it is unknown whether the increased risk can be attributed to factors that develop during pregnancy or to prepregnancy predisposing factors that 
A
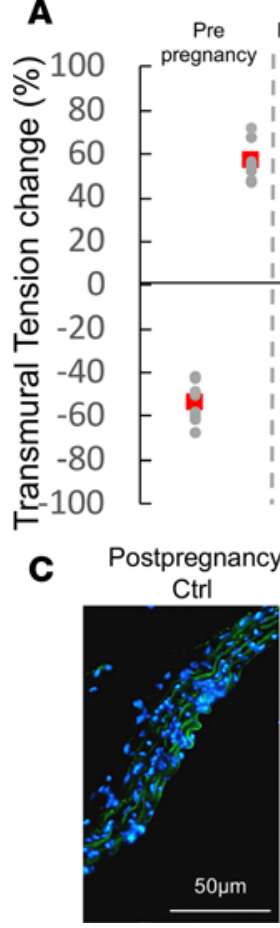

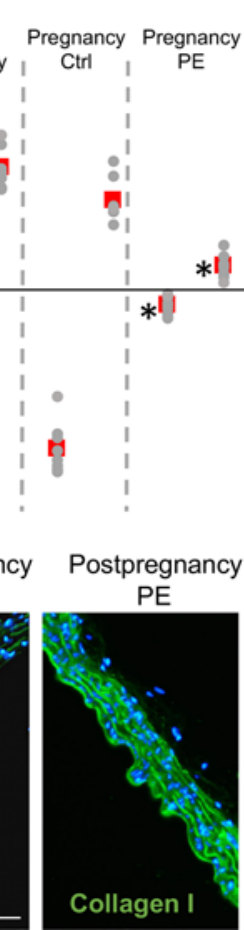

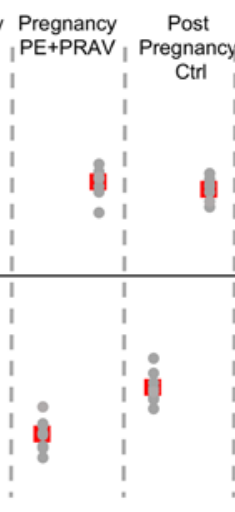

Postpregnancy PE+PRAV

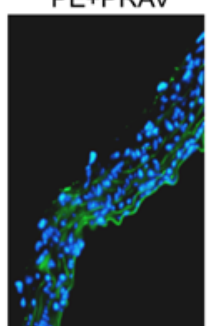

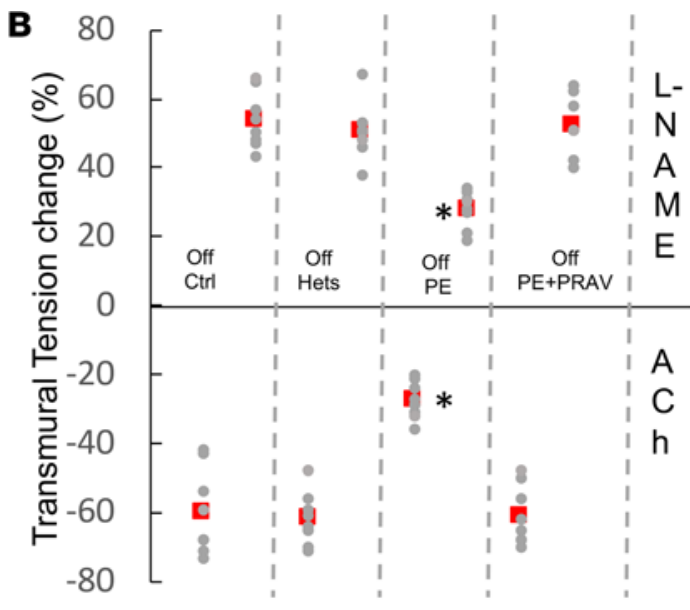

Figure 1. Endothelial function during and after preeclampsia. Wire myography results showing relaxation induced by $3 \mu \mathrm{M}$ acetylcholine (ACh) in precontracted ( $1 \mu \mathrm{M}$ phenylephrine) endothelium-intact aortic rings from mothers (A) (60 days postpartum) after normal pregnancies and pregnancies affected by preeclampsia (PE) treated and untreated with PRAV and the offspring (B) (postnatal day 30) ( $n=7-8$ mice/experimental group). Effects of NO synthesis inhibitor L-NAME $(100 \mu \mathrm{M})$ are also shown. Red squares represent the mean. The top row in $\mathbf{A}$ and $\mathbf{B}$ represents the response to L-NAME, and the bottom represents responses to ACh. Comparisons between groups were performed by ANOVA with Bonferroni's post hoc test. ${ }^{*} P<0.05$, different from control, same time point; ${ }^{\#} P<0.05$, different from prepregnancy. (C) Collagen I deposition in maternal aorta after normal pregnancies and pregnancies affected by PE (untreated and treated with PRAV) ( $n=7-8$ mice/experimental group). Increased collagen I deposition (green fluorescence), indicative of fibrosis, is observed in maternal aortas 60 days after PE. Pravastatin given during pregnancy prevented aortic fibrosis. Scale bar: $50 \mu \mathrm{m}$.

are also associated with the risk of developing PE and CVD. In addition, in humans the prepregnancy cardiovascular and renal health of the mother are not always known. The use of a mouse model in which a WT female with normal prepregnancy renal and cardiovascular health develops PE (8) will help determine if factors that develop during the preeclamptic pregnancy influence long-term maternal health.

Similar to the mothers, the offspring of pregnancies affected by PE appears to have a unique, lifetime cardiovascular risk profile that is present from early life. Children and young adults born to pregnancies complicated by PE have increased blood pressure and body mass index (9).

If $\mathrm{PE}$ is an independent risk factor that compromises the future health of the mother and offspring, strategies to modulate the release of and exposure to pathogenic factors derived from the malperfused placenta during PE can be given to prevent long-term health risks for mother and offspring. Pravastatin has been shown to improve placental hemodynamics, maternal disease, and pregnancy outcomes in the mouse model discussed herein $(8)$, other mouse models $(10,11)$, and in pilot clinical studies in women $(12-15)$. To investigate if factors released from the malperfused placenta during PE play a role in the pathogenesis of endothelial, cardiovascular, and renal disease in the mother and offspring after exposure to PE, PE mice were treated with pravastatin.

\section{Results}

Pravastatin given during pregnancy prevents endothelial dysfunction in the mother and offspring after PE. As previously described (8), endothelial dysfunction was observed in the PE mouse (8). Impaired endothelium-mediated relaxation in response to acetylcholine (ACh) and decreased contractile response to L-nitroarginine methyl ester (L-NAME) were observed during (15 days postconception [dpc]) and after pregnancy PE pregnancy (60 days) compared with control pregnancies and prepregnancy values (Figure $1 \mathrm{~A})$. In addition, some PE mice showed a paradoxical response to ACh (constriction in response to $\mathrm{ACh}$ ), characteristic of significant endothelial function impairment (16). 
Table 1. Serum levels of biomarkers in mothers and offspring

\begin{tabular}{|c|c|c|c|c|c|c|c|c|c|c|}
\hline & \multirow{2}{*}{$\begin{array}{c}\text { Before } \\
\text { pregnancy } \\
\text { Control }\end{array}$} & \multicolumn{3}{|c|}{ During pregnancy } & \multicolumn{3}{|c|}{ After pregnancy } & \multicolumn{3}{|c|}{ Offspring } \\
\hline & & Control & PE & PE+PRAV & Control & $\mathrm{PE}$ & $\mathrm{PE}+\mathrm{PRAV}$ & Control het & $\mathrm{PE}$ & $\mathrm{PE}+\mathrm{PRAV}$ \\
\hline PIGF (pg/ml) & $0.18 \pm 0.04$ & $0.671 \pm 0.32$ & $0.16 \pm 0.07^{A}$ & $0.71 \pm 0.11$ & $0.21 \pm 0.07$ & $0.24 \pm 0.16$ & $0.29 \pm 0.11$ & $0.22 \pm 0.06$ & $0.24 \pm 0.06$ & $0.18 \pm 0.09$ \\
\hline $\begin{array}{l}\text { sFlt-1 (ng/ } \\
\mathrm{ml})\end{array}$ & $6.75 \pm 1.35$ & $8.57 \pm 2.1$ & $17.89 \pm 2.46$ & $9.13 \pm 1.79$ & $7.54 \pm 1.27$ & $11.23 \pm 2.13$ & $7.86 \pm 1.14$ & $6.56 \pm 1.13$ & $6.14 \pm 0.98$ & $6.45 \pm 0.76$ \\
\hline $\begin{array}{l}\text { Endothl (pg/ } \\
\mathrm{ml} \text { ) }\end{array}$ & $50 \pm 12$ & $39 \pm 9$ & $129 \pm 31^{A}$ & $43.3 \pm 7$ & $45 \pm 9$ & $87 \pm 7^{A}$ & $51 \pm 9$ & $44 \pm 6$ & $67 \pm 7^{A}$ & $47 \pm 12$ \\
\hline $\begin{array}{l}\text { 8-STAT (pg/ } \\
\mathrm{ml} \text { ) }\end{array}$ & $760 \pm 203$ & $640 \pm 187$ & $3,275 \pm 490^{A}$ & $583 \pm 28$ & $747 \pm 272$ & $\begin{array}{c}2,487 \pm \\
1,183^{A}\end{array}$ & $681 \pm 114$ & $654 \pm 276$ & $798 \pm 157$ & $586 \pm 37$ \\
\hline $\mathrm{NO}(\mu \mathrm{mol} / \mathrm{I})$ & $75 \pm 18$ & $131 \pm 28^{B}$ & $45 \pm 17^{A}$ & $112 \pm 9$ & $85 \pm 21$ & $4,312^{A}$ & $100 \pm 15$ & $76 \pm 21$ & $73 \pm 32$ & $69 \pm 9$ \\
\hline $\begin{array}{l}\text { Insulin (ng/ } \\
\mathrm{ml} \text { ) }\end{array}$ & $0.18 \pm 0.06$ & $0.2 \pm 0.02$ & $0.83 \pm 0.27^{A}$ & $0.31 \pm 0.07$ & $0.33 \pm 0.08$ & $1.32 \pm 0.8^{A}$ & 0.260 .1 & 0.170 .15 & $0.57 \pm 0.11^{A}$ & $0.22 \pm 0.09$ \\
\hline IL-6 (ng/ml) & $8 \pm 2$ & $11 \pm 3$ & $53 \pm 7^{A}$ & $15 \pm 4$ & $12 \pm 3$ & $29 \pm 4^{A}$ & $9 \pm 3$ & $10 \pm 3$ & $13 \pm 4$ & $8 \pm 3$ \\
\hline \multicolumn{11}{|c|}{$\begin{array}{l}\text { Serum levels of angiogenic factor placental growth factor (PIGF), antiangiogenic factor sFlt-1, marker of vascular dysfunction endothelin I (EndothI), } \\
\text { markers of metabolic syndrome leptin and insulin, vasorelaxant and antiinflammatory NO, vasoconstrictors } 8 \text {-isoprostane (8-STAT) and angiotensin } \\
\text { II (AngII), and proinflammatory mediators IL-6 and C5a prepregnancy, during and after pregnancy in mother and offspring in control, PE, and PE+PRAV } \\
\text { mice. Eight to ten mice were studied in each experimental group. Comparisons between groups were performed by } 1 \text {-way ANOVA with Dunnett's post } \\
\text { hoc test within group and with Bonferroni's post hoc test between groups. }{ }^{A} P<0.05 \text {, different from control at the same time; }{ }^{B} P<0.05 \text {, different from } \\
\text { prepregnancy values. }\end{array}$} \\
\hline
\end{tabular}

Increased levels of endothelin I — indicative of endothelial damage - were observed in maternal sera during and after preeclamptic pregnancies (17) (Table 1). Increased levels of vasoconstrictor 8-isoprostane and diminished levels of vasodilator NO were observed during and after PE pregnancies compared with control pregnancies (Table 1). While levels of angiotensin II (AngII) were not different during pregnancy between PE mice and control mice, increased levels of AngII were observed after exposure to PE. Aortas from mothers after a preeclamptic pregnancy showed increased deposition of collagen I, indicative of aortic stiffness, compared with mothers with control pregnancies (intensity of the staining: postpregnancy control, $15.04 \pm 2.59 \mathrm{AU}$; postpregnancy $\mathrm{PE}, 35.85 \pm 3.6 \mathrm{AU}$, different from control; postpregnancy $\mathrm{PE}+\mathrm{PRAV}, 13.7 \pm 3.1 \mathrm{AU} ;$ F-ratio, 70.637; $P<0.01$ ) (Figure 1C).

Next, we investigated if pravastatin given during pregnancy prevented the abnormal endothelial function observed after exposure to PE. Preserved endothelial function was observed after pregnancy in $\mathrm{PE}$ mice treated with pravastatin ( $\mathrm{PE}+\mathrm{PRAV}$ mice) (Figure $1 \mathrm{~A})$. Administration of pravastatin to control pregnant mice did not affect endothelial function compared with untreated pregnant mice $(n=6, \mathrm{ACh}=$ $-79 \% \pm 7 \%$, L-NAME $=39 \% \pm 5 \%$ ). In addition, pravastatin prevented collagen accumulation in aortas of mothers after PE (Figure 1C). In line with the normal endothelial function observed after pregnancy in $\mathrm{PE}+\mathrm{PRAV}$ mice, serum levels of vasoactive factors endothelin I, isoprostane STAT-8, and NO were similar to those of control mice in these mice (Table 1).

The offspring from preeclamptic pregnancies, both females and males, also showed endothelial dysfunction (Figure 1B). Aortic rings from the offspring of PE mothers showed impaired vasodilation in response to $\mathrm{ACh}$ and decreased contraction in response to L-NAME when compared with the offspring of normal pregnancies (Figure 1B). Interestingly, the offspring of PE mothers treated with pravastatin during pregnancy showed a vasorelaxant response to $\mathrm{ACh}$ and vasoconstriction comparable to that of controls (Figure 1B), suggesting that cardiovascular abnormalities in the offspring might have their origin during pregnancy.

Hypertension is observed in mother and offspring after PE. Since endothelial dysfunction and hypertension are integrally related in respect to pathophysiologic mechanisms, we investigated the presence of hypertension after PE in the mother and offspring. As previously published (8), mean arterial pressure (MAP) 
A

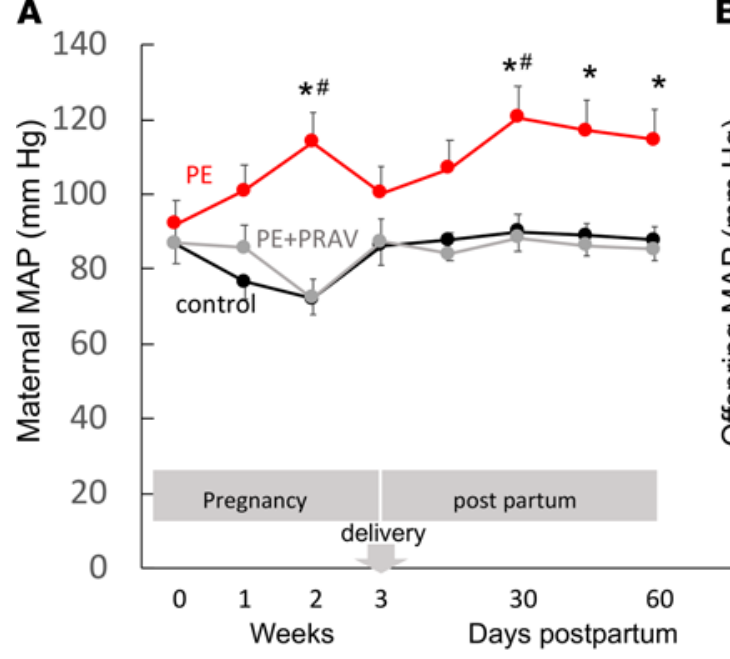

B

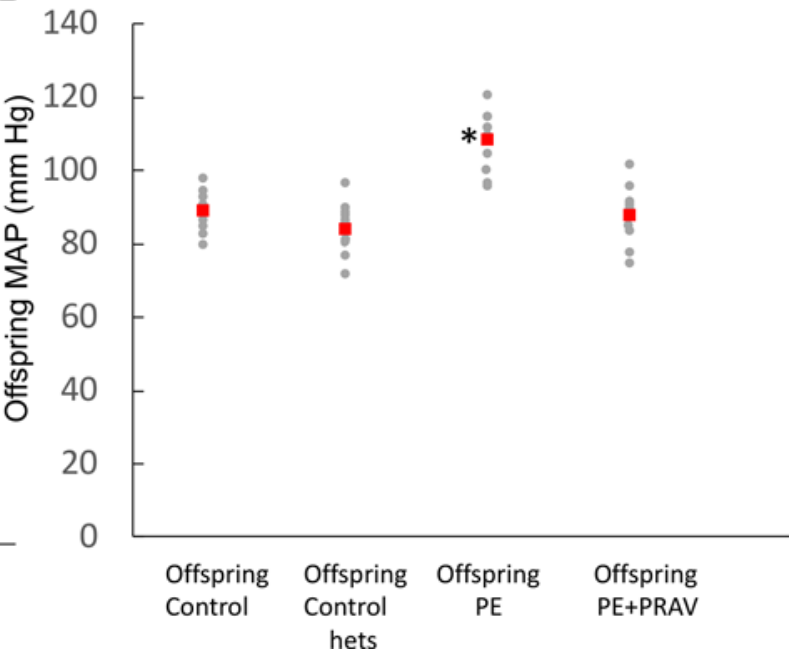

Figure 2. Mean arterial blood pressure during and after preeclampsia. (A) Mean arterial pressure (MAP) measured prior to mating, during pregnancy (first, second, and third week) and during postpartum (15, 30, 45 and 60 days) in PE mice, control mice, and PE mice treated with pravastatin (PE+PRAV). Comparisons between groups were performed by 1-way ANOVA with Bonferroni's post hoc test between groups. ${ }^{*}<0.05$, different from control mating at same time; ${ }^{\#} P<0.05$, different from previous time point. Six to eight mice were studied in each experimental group. (B) MAP in the offspring of control mice, $P E$ mice, and $\mathrm{PE}+\mathrm{PRAV}$ mice. MAP values measured in heterozygote $\mathrm{C}^{\mathrm{C}} \mathrm{q}^{+-}$offspring from mice that did not develop PE (offspring control hets) are also shown. Red squares represent the mean. ${ }^{*} P<0.05$, different from control. Ten to twelve mice ( 2 offspring/mother) were studied in each experimental group.

dropped significantly in PE mice soon after delivery of the placenta. Interestingly a progressive increase in MAP was observed after delivery in the mother. A significant increase in MAP was observed at 30 days in the postpartum mice, and MAP remained increased for up to 60 days (Figure 2A). A mild hypertension was also observed in the offspring, males and females, of PE mothers 30 days after birth (P30) compared with offspring control groups (Figure 2B). Values shown in Figure 2B include both females and male offspring, as both were shown to be equally hypertensive. Treatment of PE mothers during pregnancy with pravastatin prevented hypertension in the mother and offspring after pregnancy (Figure 2).

Renal injury is exacerbated after PE. Abnormal renal function, characterized by an increased albumin-to-creatinine ratio (ACR) and blood urea nitrogen (BUN) levels, was observed in mothers during and after PE compared with controls (Table 2).

Immunohistochemical studies showed increased fibrin and tissue factor deposition in glomeruli during and after PE compared with control pregnancies (Figure 3A). Interestingly, increased complement C3 deposition - which was found to be increased in renal biopsies of women with PE (18) - was also observed during and after PE in the mouse model (Figure 3A). Deposition of collagen I was increased in the glomeruli from maternal kidneys after PE compared with kidneys from mothers with a previous normal pregnancy (Figure 3B), suggesting progressive fibrosis of the kidneys (19).

It has been shown that mesangial cells of glomeruli damaged by hypertension show increased expression of $\alpha$-smooth muscle actin (ASMA) (20). After PE, expression of ASMA was visualized in the mesangium of glomeruli from maternal kidneys, while no expression of ASMA was detected in normotensive mothers after normal pregnancies (Figure 3B). The offspring of mothers with PE and

Table 2. Kidney function before, during and after pregnancy

\begin{tabular}{|c|c|c|c|c|c|c|c|c|}
\hline & Before & Pregnancy & Pregnancy & Pregnancy PE & Pregnancy & After pregnancy & After pregnancy & y After pregnancy \\
\hline & pregnancy & control & control+PRAV & & PE+PRAV & control & PE & PE+PRAV \\
\hline ACR $(\mu \mathrm{g} / \mathrm{mg})$ & $85 \pm 12$ & $102 \pm 15$ & $108 \pm 8$ & $845 \pm 125^{A, B}$ & $123 \pm 31$ & $98 \pm 17$ & $365 \pm 26^{A, B}$ & $141 \pm 34$ \\
\hline BUN (mg/dl) & $21 \pm 4$ & $20 \pm 5$ & $22 \pm 4$ & $38 \pm 5^{A, B}$ & $26 \pm 5$ & $23 \pm 5$ & $41 \pm 6^{A, B}$ & $25 \pm 4$ \\
\hline
\end{tabular}

Albumin-to-creatinine ratio (ACR) and blood urea nitrogen (BUN) levels before, during, and after pregnancy in control mice and mice that develop PE (untreated and treated with pravastatin (PRAV) ( $n=8-10 /$ group). Comparisons between groups were performed by 1-way ANOVA and with Bonferroni's post hoc test. ${ }^{A} P<0.05$, different from prepregnancy values; ${ }^{B} P<0.01$, different from pregnancy values. 

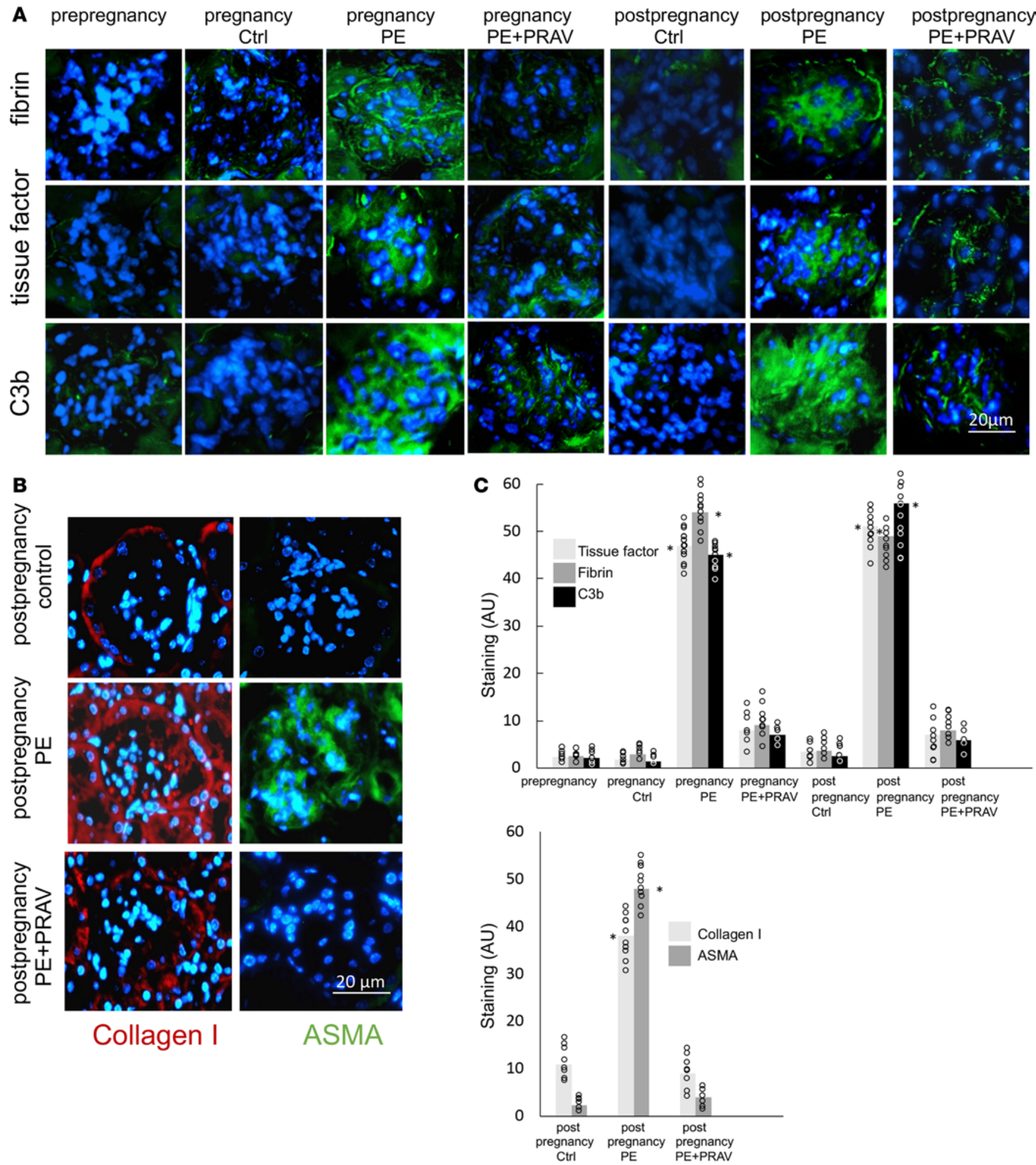

Figure 3. Glomerular damage in kidneys during and after preeclampsia. Protective effects of pravastatin. Glomerular lesions were evaluated by immunohistochemistry. The glomerular lesions observed during and after preeclampsia (PE), characterized by increased tissue factor, fibrin, and

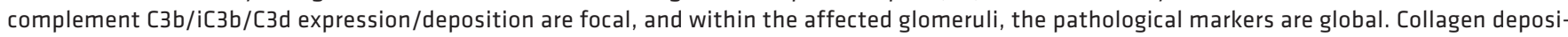
tion and $\alpha$-smooth muscle actin (ASMA) expression in the glomeruli from mothers after PE are also focal and global. Representative images of the affected glomeruli are shown. (A) Immunohistochemical (IHC) detection of tissue factor, fibrin, and complement activation product C3b/iC3b/C3d in glomeruli from maternal kidneys harvested before, during, and after pregnancy in control mice, PE mice, and PE+PRAV mice. Scale bar: $20 \mu \mathrm{m}$. Five to six images per experimental group ( $n=6-7$ mice/group) and ten glomeruli per slide were analyzed. (B) IHC detection of collagen I (red fluorescence) and ASMA (green fluorescence) staining in maternal kidneys after normal pregnancies (control) and preeclamptic pregnancies (untreated $[P E]$ and treated with pravastatin [PE+PRAV]). Scale bar: $50 \mu \mathrm{m}$. Five to six images per experimental group $(n=6-7$ mice/group) and ten glomeruli per slide were analyzed. (C) Quantification of IHC staining of tissue factor, fibrin, and C3b/iC3b/C3d and collagen I and ASMA using ImageJ. Five to 
six images per experimental group ( $n=6-7$ mice/group) and ten glomeruli per slide were analyzed. Red squares represent the mean. Comparisons between groups were performed by 1-way ANOVA with Bonferroni's post hoc test. ${ }^{*} P<0.01$, statistically different from control at same time.

control pregnancies did not show glomerular damage or abnormal renal function at P30.

Treatment with pravastatin during pregnancy prevented glomerular damage during and after pregnancy in PE mice (Figure 3A). Glomerular fibrin, tissue factor, and complement C3 deposition during and after pregnancy in PE+PRAV mice were not different from mice with normal pregnancies (Figure 3A). Pravastatin, given during pregnancy, also prevented renal deposition of collagen I and expression of ASMA in PE mice after pregnancy (Figure 3, B and C).

Normal albumin excretion, indicative of preserved glomerular structure, was observed in PE+PRAV mice (Table 2). BUN levels after pregnancy in PE+PRAV mice were not different from control mice (Table 2).

Proinflammatory and vasoactive placental-derived factors. $\mathrm{PE}$ is considered a syndrome caused by excessive inflammation (21). In this line, increased levels of proinflammatory mediators C5a and IL-6 were observed in the mothers during and after PE (Table 1). In addition, a slight increase in complement split product C5a was observed in the offspring of PE mice, while IL-6 levels in the PE offspring were not different from those of the offspring from control mice (Table 1). Treatment with pravastatin during pregnancy prevented C5a and IL-6 increase in PE mice during and after pregnancy and normalized C5a levels in the offspring of PE mice (Table 1).

Increased levels of vasoactive mediators, endothelin I and 8-isoprostane, were observed during and after pregnancy in PE mice. AngII levels during pregnancy were not different from those of control mice but were higher than those of control mice during the postpartum period (Table 1).

As seen in humans, lower levels of angiogenic factor placental growth factor (P1GF) and increased levels of soluble fms-like tyrosine kinase 1 (sFlt-1) were observed during pregnancy in the mouse model of PE compared with control mice. P1GF levels after pregnancy were not different between mice that experienced $\mathrm{PE}$ and mice with normal pregnancies. Interestingly, increased sFlt-1 levels were observed in postpartum $\mathrm{PE}$ mice (Table 1). Treatment with pravastatin during pregnancy restored the angiogenic balance (P1GF and sFLt1 levels) during and after pregnancy in PE mice. Increased concentrations of leptin and insulin, indicative of metabolic disease, were detected in maternal sera during and after PE and in the offspring, females and males, of PE mice compared with control mice (Table 1). Furthermore, the body weight of the mothers after PE was significantly higher compared with that of control mice (body weight: $32.5 \pm 1.7$ g vs. $26.8 \pm 1.9 \mathrm{~g}, n=6-8$ /group, $P<0.01)$. In parallel, significant amounts of intra-abdominal fat were observed in mice after PE compared with mice that had a normal pregnancy $(2.40 \pm 0.90 \mathrm{~g}$ vs. $0.65 \pm 0.31$ g, $P<0.01, n=6-8$ /group)

Treatment with pravastatin during pregnancy diminished insulin and leptin levels in mothers during and after PE and in the offspring (Table 1). Less intra-abdominal fat $(0.82 \pm 0.27 \mathrm{~g})$ and decreased body weight $(28.1 \pm 0.8 \mathrm{~g})$ were observed in the PE mothers treated with pravastatin compared with untreated $\mathrm{PE}$ mice after pregnancy.

Abnormal left ventricular architecture in the maternal hearts after preeclamptic pregnancies. Since left ventricular (LV) hypertrophy can develop secondary to hemodynamic factors, accompanying hypertension and inflammation may also contribute to ventricular remodeling, the next aim was to assess cardiac structure and function by ultrasound imaging. Changes in global LV structure were observed in the mothers after preeclamptic pregnancies compared with control pregnancies. Increased LV wall thickness and LV mass, together with increased stroke volumes and end diastolic and systolic volumes, were measured in the maternal hearts after PE (Figure 4 and Table 3). Immunohistochemical studies demonstrated increased collagen I deposition and increased metalloproteinase 9 expression in the LVs from mothers after a pregnancy complicated by PE compared with mice that had uneventful pregnancies (Figure 5). The structural changes observed after $\mathrm{PE}$ are indicative of myocardial fibrosis during LV remodeling. A significant increase in cell size was also observed in cardiomyocytes in mothers after PE (Figure 5D), suggesting that both cell hypertrophy and fibrosis contribute to ventricular hypertrophy. Interestingly, pravastatin treatment during PE prevented cardiomyocytes hypertrophy, collagen I deposition, and MMP-9 expression in the LV, thus preventing LV fibrosis and hypertrophy after PE (Figure 5).

Microglia activation in the brains of offspring born to preeclamptic mothers. Immunohistochemical studies showed increased staining for Iba-1 - a marker of microglia activation — in the P30 brains of offspring 


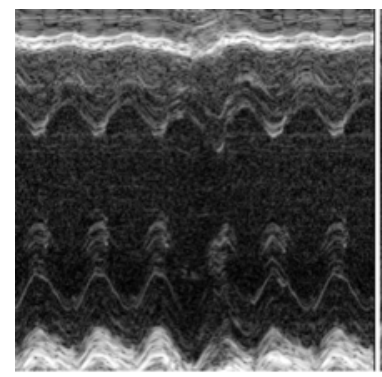

after control

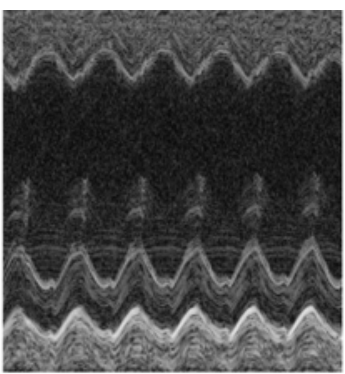

after PE

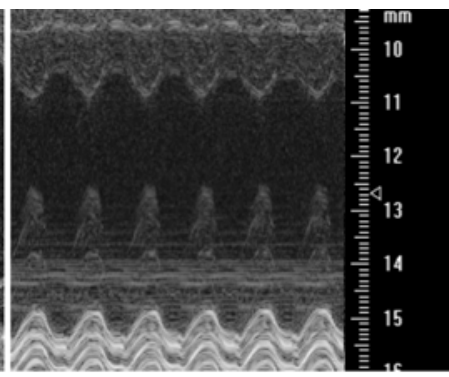

after PE+PRAV

Figure 4. Transthoracic views of the maternal hearts 60 days after pregnancy, acquired using Vevo770 ultrasound system. Representative M-mode images of the left ventricle (LV) of maternal hearts after a normal pregnancy (control mice), preeclamptic pregnancy (PE mice), and preeclamptic pregnancy treated with pravastatin (PE+PRAV). The $y$ axis represents the distance $(\mathrm{mm})$ from the transducer; time is shown on the $x$ axis. The M-mode images show the LV anterior wall, LV chamber, and LV posterior wall throughout diastole and systole. Echogenic peaks visible along the PW during systole represent the papillary muscle entering the field of view. Images were acquired using the Vevo 770 ultrasound system (VisualSonics).

born to PE mothers compared with the offspring born to uncomplicated pregnancies (Figure 6A). Interestingly, no signs of microglia activation were observed in the brains of the 30-day-old offspring from PE mice that were treated with pravastatin during pregnancy.

In addition, a larger number of activated cells, characterized by a round shape and small thick or absent processes (Figure 6A, right inset), was observed in the microglia isolated by immune affinity purification from P10 brains of PE offspring (Figure 6B). Microglia isolated from offspring of $\mathrm{PE}+\mathrm{PRAV}$ mice showed a resting, nonactivated phenotype, (Figure 6A, left inset) comparable to that of control mice born to mothers with uneventful pregnancies (Figure 6B). Interestingly, microglia isolated from $\mathrm{PE}$ offspring displayed a proinflammatory phenotype, as determined by upregulated levels of TNF- $\alpha$ and IL-6, whereas microglia isolated from control mice and mice born to PE+PRAV mothers did not (Figure 6C). Microglia from offspring born to PE mothers treated with pravastatin did not show an increased proinflammatory response to LPS. Amounts of IL- 6 and TNF- $\alpha$ comparable to those found in microglia from the control offspring were observed in the supernatants of microglia from PE+PRAV offspring (Figure 6C).

RT-PCR studies confirmed the activated proinflammatory state observed in the microglia isolated from brains from the PE offspring. Increased gene expression for proinflammatory cytokines IL-6, TNF- $\alpha$, TGF- $\beta$, and IL- $1 \beta$ was observed in the microglia isolated from PE offspring compared with the microglia isolated from the control offspring (Figure 7A). Microglia isolated from the offspring born to $\mathrm{PE}$ mothers treated with pravastatin showed gene expression for proinflammatory cytokines comparable to the control group, indicative of resting, not activated, microglia (Figure 7B).

\section{Table 3. Echocardiography data}

\begin{tabular}{|c|c|c|c|c|c|c|c|c|c|}
\hline $\begin{array}{l}\text { Maternal heart } \\
60 \text { days after } \\
\text { pregnancy }\end{array}$ & $\begin{array}{l}\text { LV internal } \\
\text { diameter } \\
\text { (diastole) } \\
\text { (mm) }\end{array}$ & $\begin{array}{c}\text { LV internal } \\
\text { diameter } \\
\text { (systole) }(\mathrm{mm})\end{array}$ & $\begin{array}{l}\text { Stroke volume } \\
\text { ( } \mu \mathrm{l})\end{array}$ & $\begin{array}{c}\text { Ejection } \\
\text { fraction }(\%)\end{array}$ & $\begin{array}{c}\text { Fractional } \\
\text { shortening (\%) }\end{array}$ & $\begin{array}{l}\text { Cardiac output } \\
\text { (ml/min) }\end{array}$ & $\begin{array}{c}\text { Volume } \\
\text { (diastole) }(\mu \mathrm{l})\end{array}$ & $\begin{array}{c}\text { Volume } \\
\text { (systole) }(\mu \mathrm{l})\end{array}$ & $\begin{array}{l}\text { LV mass (AW) } \\
\text { corrected (mg) }\end{array}$ \\
\hline $\mathrm{PE}(n=5)$ & $4.41 \pm 0.07$ & $3.15 \pm 0.10$ & $62.31 \pm 8.78^{A}$ & $60.32 \pm 4.92$ & $32.27 \pm 3.47$ & $29.50 \pm 3.30$ & $87.84 \pm 3.30^{A}$ & $39.75 \pm 3.12^{A}$ & $\begin{array}{c}149.34 \pm \\
15.04^{\mathrm{A}}\end{array}$ \\
\hline $\begin{array}{l}\text { PE+PRAV } \\
(n=5)\end{array}$ & $3.97 \pm 0.19$ & $37.6 \pm 0.22$ & $44.02 \pm 4.51$ & $61.10 \pm 3.82$ & $32.74 \pm 2.65$ & $23.74 \pm 2.65$ & $69.52 \pm 14.8$ & $28.01 \pm 5.80$ & $127.05 \pm 26.08$ \\
\hline$P$ value & 0.0063 & 0.02934 & 0.00038 & $>0.05$ & $>0.05$ & $>0.05$ & 0.012 & 0.0247 & 0.0286 \\
\hline
\end{tabular}

Baseline conventional 2-dimensional echocardiographic measurements in maternal hearts after a normal pregnancy and pregnancy complicated by PE (with and without pravastatin treatment). Echocardiography measures were obtained from a short-axis view at the level of the papillary muscle. Values are mean \pm SD ( $n=5-7$ mice/experimental group). Comparisons between groups were performed by 1-way ANOVA, indicated by F-ratios with Bonferroni's post hoc test. ${ }^{A} P<0.05$, different from control. AW, anterior wall. 
A After control pregnancy

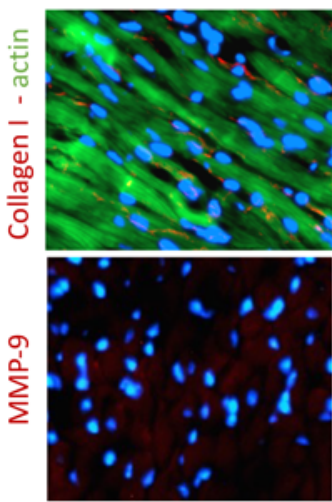

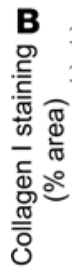

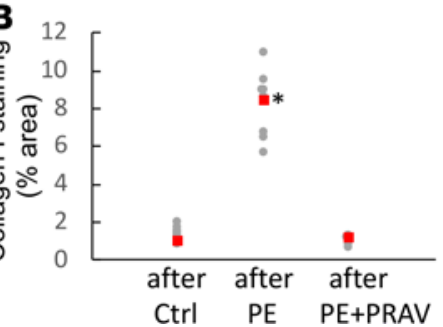

After pregnancy PE
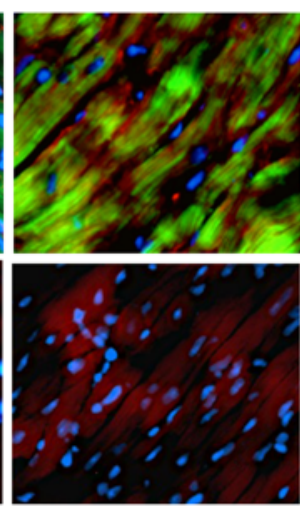

C

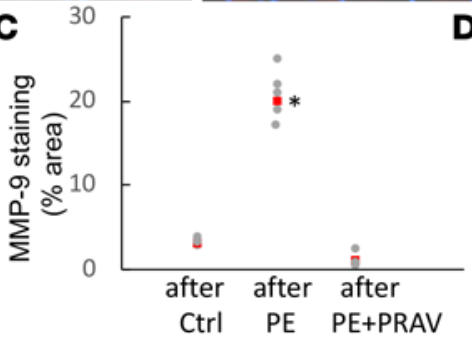

After pregnancy PE+PRAV
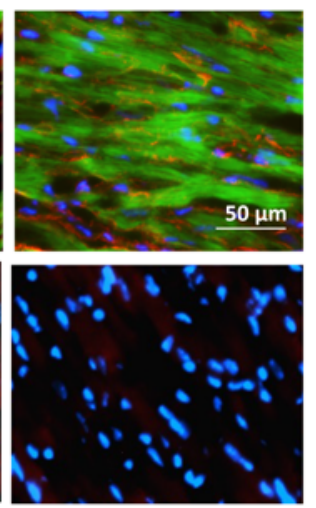

D

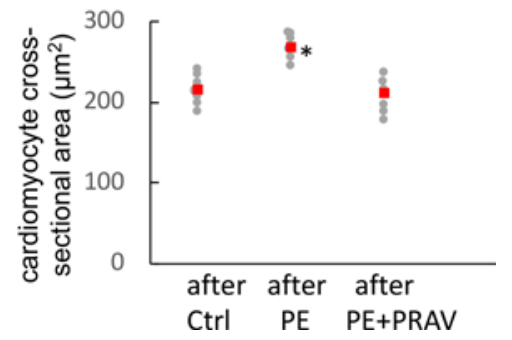

Figure 5. Representative microphotographs of the left ventricle in maternal hearts after a normal pregnancy and pregnancies complicated by preeclampsia (with and without pravastatin treatment). (A) Increased collagen deposition (red fluorescence, top panels), indicative of fibrosis, and MMP- 9 (red fluorescence, bottom panels), indicative of tissue remodeling, are observed in the maternal heart 60 days after preeclampsia. Green fluorescence in top panels indicates F-actin within the cardiomyocytes. Pravastatin prevented LV fibrosis/remodeling. Ten slides per experimental group (5-7 mice/experimental group) were analyzed. Scale bar: $50 \mu \mathrm{m}$. (B and C) Quantification of staining for collagen I and MMP-9 expressed as a percentage of the tissue area. Ten slides per experimental group were analyzed for collagen content and MMP-9 expression. Raw data and mean \pm SD are shown. Comparisons between groups were performed by 1-way ANOVA with Bonferroni's post hoc test. ${ }^{*} P<0.01$, different from control. (D) Quantification of cross-sectional area of cardiomyocytes in left ventricles. Red squares represent the mean. Areas of 50 myocytes from 5 randomly selected microscope fields from the LV posterior wall from each experimental group ( $n=5-7$ mice/experimental group) were averaged to represent the myocyte area. ${ }^{*} P<0.05$, different from control.

\section{Discussion}

CVD represents one-third of all deaths among women (1). Effective strategies to identifying and preventing gender-specific risk factors remain paramount. It has been suggested that PE constitutes a risk factor for CVD after pregnancy. In this study, using a mouse model, we demonstrated that factors that develop during $\mathrm{PE}$ have long-term health effects on the cardiovascular system of the mother and offspring, independent of prepregnancy risk factors. Endothelial dysfunction, hypertension, and renal damage observed in the mouse model during PE were also detected after PE, confirming that PE is not a transient disease. While hypertension in PE mice was ameliorated after delivery, probably due to the regression of hypervolemia and cessation of the hormonal activation that causes sodium and water retention during pregnancy, hypertension was detected again during the postpartum.

Increased levels of sFlt-1 measured during and after PE might contribute to the glomerular dysfunction observed in PE mice. Interestingly, our results show that the placenta is not the only source of sFlt- 1 , as sFlt-1 levels were also elevated after PE, suggesting that the injured endothelium might contribute to the production of this antiangiogenic factor. Renal injury observed during PE, further exacerbated after pregnancy, and signs of renal fibrosis were observed. Deposition of pathological matrix, such as collagen within the glomerular tufts, is an important factor in the amplification of kidney injury and acceleration of nephron demise. Furthermore, ASMA was detected in the mesangium of maternal kidneys after PE. Glomerular expression of ASMA is a sign of glomerular injury that has been described in response to hypertension and AngII $(20,22)$. In this line, hypertension and increased levels of AngII were observed in mice after PE compared with control mice with normal pregnancies.

The special relationship and the interdependence of the kidneys and the heart is well recognized. Kidney injury and hypertension that were observed during and after pregnancy might affect cardiac function after pregnancy. During pregnancy, the heart develops physiologically reversible LV hypertrophy - not associated with fibrosis and detrimental long-term effects on cardiac function — as a 
A
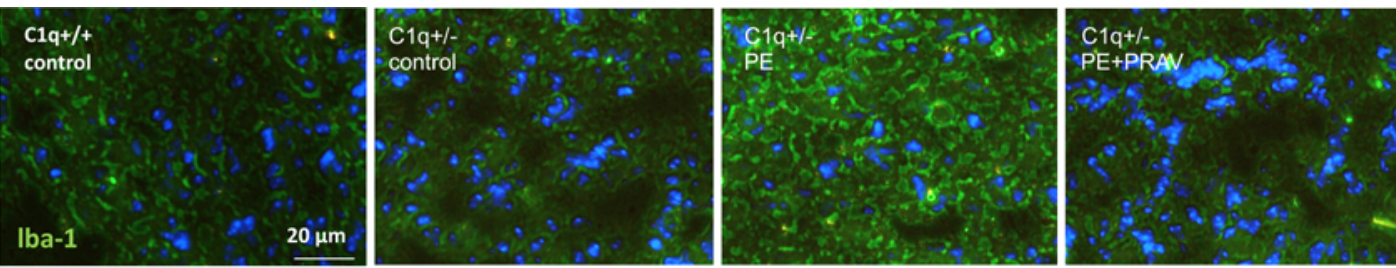

$\mathbf{B}$

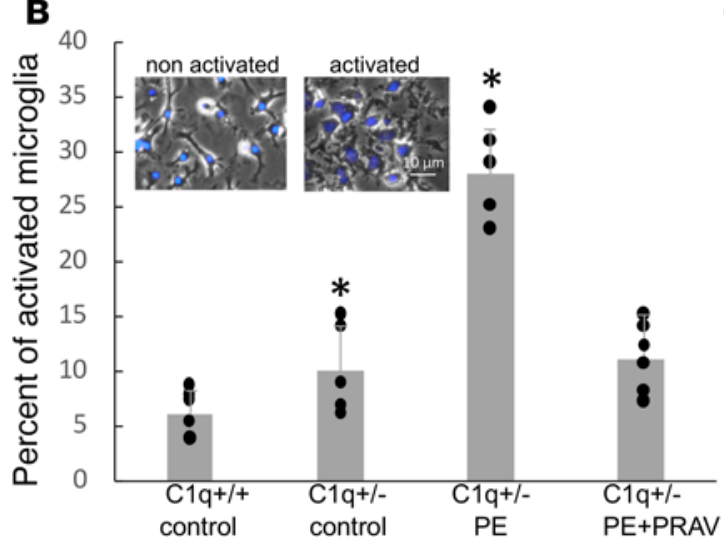

c

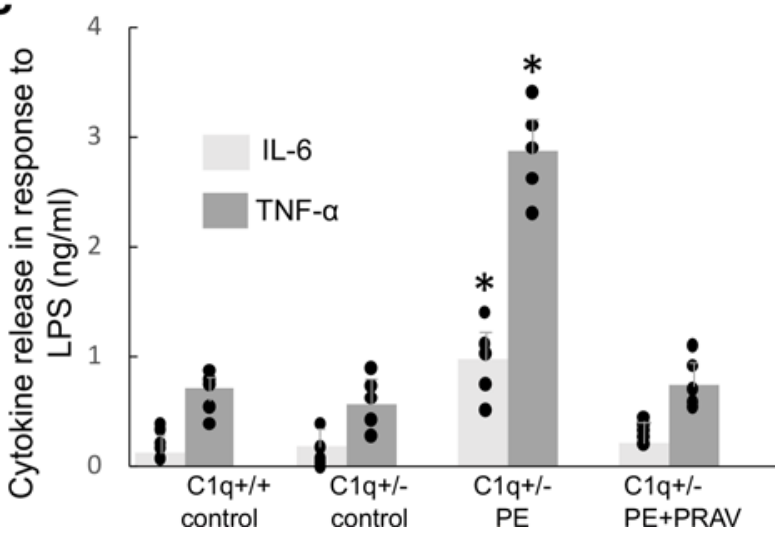

Figure 6. Signs of microglia activation in the brains of offspring born to preeclampsia mothers. (A) Expression of ionized calcium-binding adaptor molecule 1 (Iba-1) - a marker of activated microglia - in neonatal brains (P30) from pups born to control mothers and mothers with PE (untreated and treated with pravastatin). Increased Iba-1 staining is observed in the brains of offspring born to PE mothers. Ten slides per experimental group (5-7 mice/experimental group) were analyzed. Scale bar: $20 \mu \mathrm{m}$. Staining quantification was performed using Image], and comparisons between groups were performed by 1-way ANOVA with Bonferroni's post hoc test $\left(\mathrm{C}^{1 q^{+/+}}: 9.8 \pm 2.1 \mathrm{AU}, \mathrm{C1q}^{+/-}\right.$: [control] $12 \pm 2.3 \mathrm{AU}, \mathrm{C1q}^{+/-}[\mathrm{PE}]: 53^{*} \pm 14 \mathrm{AU}[\mathrm{P}<0.05], \mathrm{C} 1 \mathrm{q}^{+/-}[\mathrm{PE}+\mathrm{PRAV}]: 11 \pm 3.2$ AU; $P<0.05$, different from $\left(1 q^{+/+}\right)$. (B) The percentage of activated cells in immunopurified microglia isolated from P10 pups born to control mothers and $\mathrm{PE}$ mothers (untreated and treated with pravastatin during pregnancy). Four brains were dissected from the same litter and pooled for microglia isolation ( $n=5-6$ litters). Resting, nonactivated cells were identified by a small soma and ramified long thin processes (B, left inset). Activated microglia were less ramified and oval amoeboid-shaped cells (B, right inset). Scale bar: $10 \mu \mathrm{m}$. The number of activated microglia expressed as a percentage of the total number of microglia was used as a measure for microglial activation. (C) To evaluate the proinflammatory phenotype in microglia, microglial cells isolated from pups born to mothers subjected to normal and abnormal pregnancies (untreated and treated with pravastatin) were exposed to $1 \mu \mathrm{g} / \mathrm{ml} \mathrm{LPS} \mathrm{in} \mathrm{the} \mathrm{culture}$ medium for 24 hours. The supernatants were collected and measured for the presence of proinflammatory cytokines TNF- $\alpha$ and IL-6 by ELISA. Microglia isolated from pups born to untreated PE mothers showed an increased release of TNF- $\alpha$ and IL- 6 in response to LPS indicative of a proinflammatory M1 phenotype. (B and C) Four brains were dissected from pups from the same litter and pooled for microglia isolation ( $n=5-6$ litters/ experimental group). Comparisons between groups were performed by 1-way ANOVA with Bonferroni's post hoc test. ${ }^{*} P<0.01$, different from control groups.

compensatory response, allowing the heart to maintain its pumping capacity (23). In contrast, maternal LV hypertrophy in mice after PE was not transient and was associated with ventricular remodeling that may lead to overt failure. The architectural changes observed in the LV and aorta might be caused by high blood pressure (BP). Various hypertensive stimuli lead to increased collagen deposition in large arteries, exacerbating BP elevation and end-organ damage. In this connection, increased collagen deposition was observed in the aortas of mice after PE. This is in keeping with a study reporting that women with early-onset PE, and thus, exposure to hypertension for longer periods, develop more significant cardiac remodeling than the ones that develop late PE (24). In addition, there is evidence to support an association of mild renal insufficiency with ventricular remodeling and fibrosis (25). AngII might also contribute to the development of fibrosis. AngII activates fibroblasts, increasing the synthesis of extracellular matrix proteins (26). In this line, increased levels of AngII correlated with the presence of renal, aortic, and cardiac fibrosis after PE.

The sustained maternal inflammatory response observed after PE might also affect the cardiovascular and renal structure and function. Innate and adaptive immune responses have an essential role in the development and progression of many CVDs (27). A recent study associated proinflammatory cytokine IL-6 with the pathogenesis of both LV hypertrophy and renal fibrosis (28, 29). Complement factor $\mathrm{C} 5 \mathrm{a}$ is an important mediator in acute kidney injury and fibrosis and ventricular stiffness in rat models (30). In addition, C5a has also been related to the pathogenesis of hypertension in a rat model of PE (31). Furthermore, leptin and insulin — markers of metabolic disease - confer an increased risk for LV remodeling in humans $(32,33)$. 
A

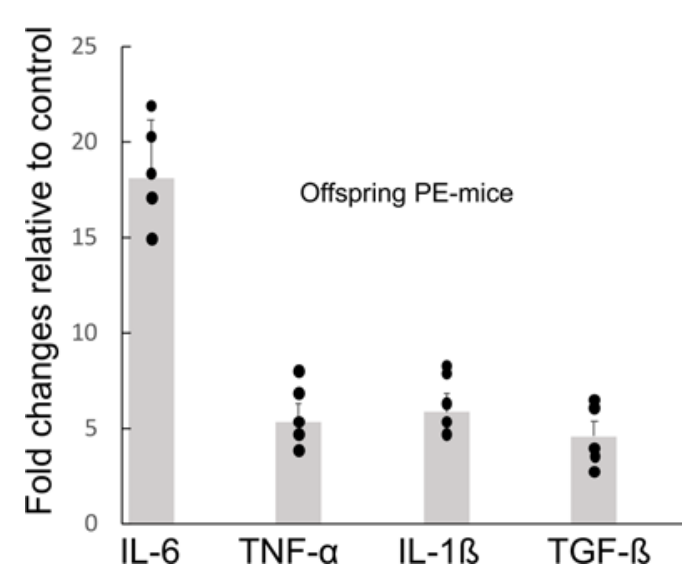

B

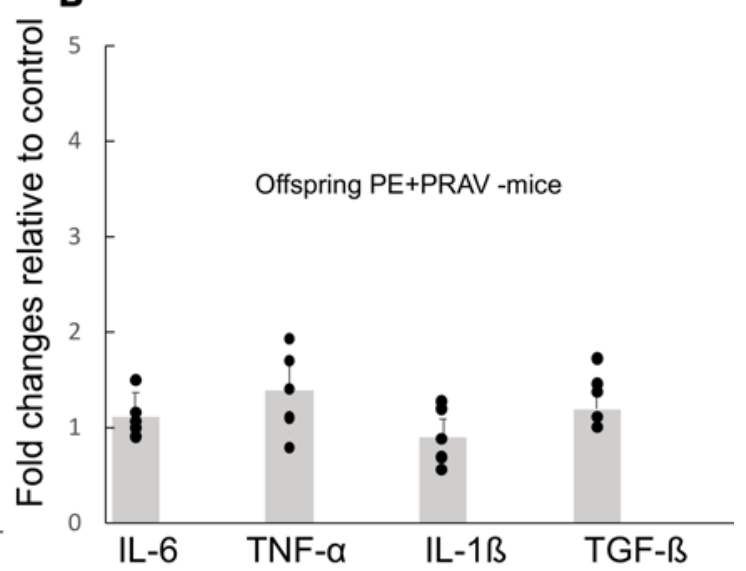

Figure 7. Proinflammatory cytokines gene expression is increased in microglia isolated from the offspring of PE mothers. Proinflammatory cytokine gene expression in immunopurified isolated microglia was measured using real-time PCR. Gapdh was used to normalize the quantitative experiments based on prior reference gene suitability testing. The specific ratio between the gene of interest and the reference gene was calculated for each sample. (A) Results are expressed as the ratio between gene expression in the PE offspring and control offspring. Increased expression of proinflammatory cytokine genes IL-6, TNF- $\alpha$, IL-1 $\beta$, and TGF- $\beta$ was observed in microglia isolated from offspring born to PE mothers (PE offspring) compared with control offspring ( $\mathrm{Clq}^{+/-}$born to $\mathrm{C} 1 \mathrm{q}$-sufficient fathers and thus not subjected to $\mathrm{PE}$ ). (B) Results are expressed as the ratio between gene expression in the PE+PRAV offspring and control offspring. Pravastatin prevented the increased expression of proinflammatory cytokines observed in the PE offspring. Four brains were dissected from pups from the same litter and pooled for microglia isolation and further RNA isolation ( $n=5-6$ litters/ experimental group). Comparisons between groups were performed by 1-way ANOVA with Bonferroni's post hoc test.

There is growing evidence regarding the transplacental passage of maternal-derived mediators to the fetus. Development of the fetus is highly sensitive to alterations in the intrauterine environment, and exposure to these factors in utero during PE might affect the development of the cardiovascular and the nervous system in the fetus. Indeed, the offspring of PE mice developed endothelial dysfunction, hypertension, and signs of metabolic disease. This is in support of the "developmental origins of adult disease" hypothesis, which posits that a significant portion of the risk for adult conditions is determined by exposures taking place in the prenatal period (34). In parallel, our data suggest that placental-derived factors released during $\mathrm{PE}$, such as 8-isoprostane, endothelin I, sFlt-1, NO, C5a, and IL-6, might affect the fetal development leading to CVD and metabolic syndrome in the offspring. Exposure in utero to placental pathogenic mediators might affect fetal neurodevelopment, leading to microglia activation and stimulation of the sympathetic nervous system, leading to neurogenic hypertension in the offspring (35). Microglia are macrophages, and as such they express $\mathrm{C} 5 \mathrm{a}$ receptors. Increased microglia activation might be caused by the increased levels of C5a observed during and after PE in mother and offspring.

In addition, placental dysfunction/mal perfusion, observed during PE, might exacerbate abnormal fetal development by failing to provide adequate amounts of oxygen and nutrients to the fetus. In addition, intrauterine growth restriction, observed in the PE model, might also have a direct effect on organ development, such as the kidney and the heart, contributing to the increased cardiovascular risk observed in the offspring exposed to PE (36).

Our studies suggest that the mediators that contribute to cardiovascular and renal disease after PE in the mother and offspring might originate from the placenta. Indeed, synthesis of the potential mediators in endothelial/cardiovascular and renal dysfunction identified in this study, such as 8-isoprostane, endothelin I, NO, C5a, and IL-6, has been demonstrated in the placenta. Even more, changes in their production have been associated with placental insufficiency, a hallmark of PE (37-40). Furthermore, pravastatin, by preventing placental insufficiency, inhibited the release of these pathogenic factors that compromise the cardiovascular health of mother and offspring. These pathogenic factors that affect endothelial function might also be released from the dysfunctional endothelium and damaged kidney, leading to an amplification cycle and perpetuation of cardiovascular and renal disease in the absence of the placenta. Placental-derived and/or endothelial-derived exosomes, which increase in sera from preeclamptic pregnancies can also influence endothelial and renal function and cardiac structure $(41,42)$. Signaling molecules found in the exosomes, together with the mediators identified in our study might 
interact with immune cells and modulate the innate immune responses affecting the development, progression, and perpetuation of cardiovascular and renal structural changes that may lead to renal and cardiac disease after pregnancy (43).

In agreement with the fetal origins of adult disorders theory, our study shows a long-term health compromise - hypertension and signs of metabolic disease - in the offspring of PE mice. Interestingly, signs of metabolic syndrome were also described in the offspring of mice with sFlt-1-induced PE and rats with surgically induced placental ischemia $(44,45)$, supporting the notion that exposure to an adverse uterine environment during PE affects the long-term health of the offspring. Provocatively, the offspring of $\mathrm{PE}+\mathrm{PRAV}$ mice were normotensive and did not show signs of metabolic disease like the offspring born to untreated PE mothers. In agreement with our observations that pravastatin treatment during pregnancy prevented metabolic disease in the offspring, a recent study has also shown that pravastatin treatment reversed abnormal glucose levels in the offspring in a mouse model of PE induced by systemic overexpression of sFlt-1 (46).

Factors released during PE, most likely from the malperfused placenta, lead to renal and CVD in the mother and offspring after exposure to PE, independent of preexisting risk factors. These findings have important translational implications. While women with known preexisting factors might be followed closely during and after pregnancy for renal and cardiovascular abnormalities; our studies suggest that women with a normal prepregnancy cardiovascular and renal function that develop PE should also continue to be followed up after delivery.

There is an urgent need to identify biomarkers for long-term CVD in young women to enable preventive therapies before irreversible atherosclerosis and CVD are detected (47). In this study, several proinflammatory and vasoactive factors that can serve as biomarkers, and an early prevention strategy to halt the progression of long-term cardiovascular chronic kidney disease in the mother have been identified. These biomarkers might also help the identification of fetuses most at risk and allow for targeted surveillance and preventive therapy instigated during pregnancy. Since pravastatin therapy given during $\mathrm{PE}$ prevents long-term health compromise, it is tempting to speculate that pravastatin targets the placenta, improving its function and reversing the adverse intrauterine environment associated with PE. Pravastatin, by reversing atherosclerotic lesions frequently found in the placenta during $\mathrm{PE}$, might improve the uteroplacental circulation and prevent placental dysfunction (48). Indeed, our studies in mice and humans $(8,15)$ found that pravastatin restores placental blood flow, preventing placental insufficiency. By preventing placental insufficiency, pravastatin - a hydrophilic molecule with limited transport across the placenta (49) - might inhibit the release of pathogenic/vasoactive/inflammatory factors from the malperfused placenta and, thus, prevent the cascade of events that leads to renal and cardiac abnormalities after PE. This study builds on existing studies to demonstrate a further understanding of the beneficial properties of pravastatin in the treatment of PE. Pravastatin was shown not only to ameliorate maternal disease, but to protect the mother and offspring from cardiovascular and metabolic compromise after PE.

\section{Methods}

Animals. Mice deficient in C1q (C1qKO), fully backcrossed onto C57BL/6 (provided by Marina Botto, Imperial College, London, United Kingdom) and WT C57BL/6 mice (purchased from Charles Rivers), were used in all experiments. Eight- to 10 -week-old virgin female C57BL/6 mice were mated with 8- to 14- week-old $\mathrm{ClqKO}$ or WT males. Females were inspected daily for vaginal plugs; the first day that a vaginal plug was seen was designated as day 0 of pregnancy. Wild-type and genetically modified mice were maintained at $20^{\circ} \mathrm{C}-22^{\circ} \mathrm{C}$, with standard rodent chow available ad libitum and under a semi-natural light/ dark (12:12-hour) cycle.

Mouse model of PE. Trophoblast migration and spiral artery remodeling are essential to normal placentation and good pregnancy outcomes. Complement component C1q secreted by trophoblasts is used by these cells to invade the decidua. The role of $\mathrm{C} 1 \mathrm{q}$ in these processes has been demonstrated in mice and women $(8,50)$. Lower levels of $\mathrm{C} 1 \mathrm{q}$ in sera of pregnant women were shown to be associated with the development of PE (51). Paternally expressed genes predominate in the placenta. Thus, WT C57BL/ 6 female mice with normal cardiovascular function (52) when impregnated by a C1qKO mouse showed defective trophoblast migration/invasion and abnormal placentation, leading to placenta malperfusion. WT $\times$ C1qKO mice developed all the clinical signs observed in women with PE: systemic endothelial dysfunction, hypertension, proteinuria, angiogenic imbalance, and glomerular 
endotheliosis (8). Endothelial/cardiovascular and renal function before, during, and after pregnancy were investigated in this model that closely resembles the clinical human scenario.

Experimental groups. C57BL/6 females impregnated by C1qKO constituted the PE group (PE mice). C57BL/6 females impregnated by C57BL/6 males constituted the control group (control mice). A group of $\mathrm{PE}$ mice and control mice were treated with pravastatin $(10 \mu \mathrm{g} / \mathrm{mouse}$ i.p., human equivalent dose, $20 \mathrm{mg}$ ) from 5-14 dpc (PE+PRAV mice). C1qKO females mated with C57BL/6 males, that generate heterozygote offspring $\mathrm{C}_{1} \mathrm{q}^{+-}$but do not exhibit $\mathrm{PE}(8)$, constituted the control group for the offspring studies (control heterozygous mice). Experimental groups control, PE and PE+PRAV were studied before, during pregnancy $(15 \mathrm{dpc})$ and after pregnancy (60 days postpartum). 60 days in the postpuberal phase in a mouse is equivalent to 7-8 human years (53). In the after pregnancy experimental groups the mothers were 5.5 to 6 months old.

The offspring from PE mice, PE+PRAV mice, control mice, and control heterozygous mice were studied at 30 days after birth. Four pups ( 2 females and 2 males combined) were studied in each litter.

Wire myography. Animals were killed by cervical dislocation. Fat inside the abdominal cavity was dissected and weighed. Aortas were removed and placed into ice-cold physiological salt solution (PSS) as previously described (54). Wire myography was used for the in vitro physiological study of blood vessels (54). The thoracic aorta was dissected, cleaned of surrounding fat, cut into approximately 2.5-mm rings, and mounted into a chamber unit in a multiwire myograph system (610M, Danish Nyo Technology). Vessel tension data were recorded and stored on a computer using Myodaq 2.02 analysis software (Danish Nyo Technology). Vessels were then left to equilibrate in PSS and then subjected to constriction with high potassium (125 mM) solution (KPSS) as previously described (54). Following incubations with KPSS, vessels were constricted with $50 \mu 1$ of $10^{-4} \mathrm{~mol} / 1$ phenylephrine (MilliporeSigma) to induce a $80 \%$ maximum contraction in arteries. After vasoconstriction with phenylephrine, a dose-response curve of the response to ACh (10 $10^{-8}$ to $10^{-5} \mathrm{M}$, MilliporeSigma) was then used to assess endothelium-dependent vasodilation. After measuring responses to $\mathrm{ACh}$, NO synthase inhibitor NG-nitro-L-arginine methyl ester $\left(100 \mu 10^{-2} \mathrm{~mol} / 1\right.$ L-NAME; MilliporeSigma) was added to evaluate the effects of the absence of NO in the vascular tone. After the experiments, contractile ability of the blood vessels was tested. Vessels that failed to contract in response to KPSS or phenylephrine were not included in the study.

$B P$ measurements. BP was measured in the tail artery in conscious mice before, during (first, second, and third week) and after (15, 30, 45 and 60 days) normal and preeclamptic pregnancies (untreated and treated with pravastatin). BP in the offspring was measured 30 days after birth (P30). Measurements were performed using a computerized, noninvasive tail-cuff acquisition system (CODA System, Kent Scientific Corporation) (55). This method had been validated by comparison to simultaneous radiotelemetry BP measurements (56). The CODA system utilizes volume-pressure recording technology to detect changes in tail volume that correspond to systolic and diastolic pressures and calculates MAP during each measurement cycle. Our protocol consisted of 8 acclimation cycles and 8 measurement cycles daily. Unanesthetized mice were placed in plastic holders. Mouse body temperatures were monitored closely and maintained at between $34^{\circ} \mathrm{C}$ and $36^{\circ} \mathrm{C}$ using infrared heating. Readings differing by more than 10 $\mathrm{mmHg}$ were repeated after a rest period of 15-20 minutes.

Assessment of renal function. The ACR in random urine specimens (accepted alternative to 24-hour urine collections, ref. 57) was used to evaluate glomerular function before pregnancy, during pregnancy (15 days), and after pregnancy (60 days). Urinary albumin was determined by ELISA (Albuwell M, Exocell). Creatinine in urine was quantified with the Creatinine Companion kit (Exocell). BUN was measured using a colorimetric method (Urea Assay Kit, MilliporeSigma).

ELISAs and colorimetric assays. ELISA kits to determine P1GF, endothelin I, sFlt-1, and IL-6 were purchased from R\&D Systems. The ELISA kit to measure AngII and the colorimetric assay-based kit to determine NO levels were obtained from MilliporeSigma. C5a and leptin were measured in mouse serum using kits from Abcam. Insulin and STAT-8-isoprostane ELISA kits were purchased from Crystal Chem and Cayman Chemical, respectively.

Ultrasound imaging. Sixty days after normal and preeclamptic pregnancies, the dams were subjected to ultrasound imaging to assess cardiac structure and function. The mice were anesthetized with isoflurane (inhalation, 1.5\%). Hair from the abdomen and chest of the animals was removed, and ECG, respiration, and temperature were monitored throughout all scans. A Vevo 770 (VisualSonics Inc.) high-resolution preclinical ultrasound scanner was used to image the mice. An 30- $\mathrm{MHz}$ ultrasound transducer was placed 
on the surface of the chest to assess cardiac structure and function, and images and videos were recorded. Structural and functional cardiac assessment was performed using 1-dimensional M-mode echocardiography and 2-dimensional B-mode echocardiography and pulse-wave Doppler signal. Images were acquired from the parasternal long and short axis to measure cardiac wall and chamber dimensions, and Doppler waveforms were analyzed for blood flow measurements and to assess cardiac function. End-systolic and end-diastolic thickness of the interventricular septum, LV inner diameter, and LV volume were assessed to calculate the LV shortening, ejection fraction, stroke volume, and cardiac output. All images were analyzed off-line and the observer was blinded for echocardiography measurements.

Microglia isolation - CD11b antibody-coupled magnetic cell isolation. Microglia, resident immune cells in the brain, mediate neuroinflammation and modulate neuronal excitation, leading to elevated BP (58). We investigated if exposure to intrauterine inflammation during $\mathrm{PE}$ affects fetal brain development, leading to microglia activation and to neurogenic hypertension in the offspring.

Microglial cells were isolated from the brains of pups born to control and PE mothers at P10. Cells positive for $\mathrm{CD} 11 \mathrm{~b}$ (cluster of differentiation $11 \beta$, a marker for microglia), were extracted from P10 brains using the antibody-coupled magnetic bead system (MACS) following the manufacturer's recommendations (Miltenyi Biotec) (59). In brief, the olfactory bulbs and cerebella were removed, and the hemispheres were mechanically and enzymatically digested using the Neural Tissue Dissociation Kit (Miltenyi Biotec). Four brains were pooled for each sample to ensure sufficient RNA quantities. Homogenized and digested tissue was incubated with magnetic microbead-coupled anti-CD11b antibodies (Miltenyi Biotec, 130-093-636, 10 $\mu 1 / 10^{7}$ cells), and CD11b-positive cells were separated in a magnetic field. Immunopurified microglia were $>95 \%$ pure by staining with Iba1 antibody, a marker for the microglia. Microglial cells were cultured to evaluate their activation estate or frozen at $-80^{\circ} \mathrm{C}$ for RNA extraction.

Characterization of the state of microglia activation was performed by morphological evaluation (60), production of proinflammatory cytokines in response to LPS, and synthesis of proinflammatory cytokines.

Immunohistochemical staining for calcium-binding protein lba-1 was used to detect activated microglia in P30 brain tissue from pups born to control mothers and PE mothers (untreated and treated with pravastatin).

Morphological evaluation. Microglial cells were transferred onto 16-well chamber slides and cultured for 24 hours. Isolated microglia were morphologically characterized based on the activation/resting stage. Resting, nonactivated cells were identified by small soma and ramified, spread-out, thin, and long processes. In contrast, activated microglia were characterized by small, less-ramified oval-amoeboid shaped cells, with hypertrophied soma and retracted gross processes (60). The number of activated microglia, expressed as a percentage of the total number of microglia, was used as a measure for microglial activation.

IL- 6 and TNF- $\alpha$ production. Isolated microglial cells were plated at a density of $3 \times 10^{5} \mathrm{cells} / \mathrm{cm}^{2}$ using $100 \mu 1 /$ well DMEM-F12, containing 10\% (v/v) FBS and antibiotics, and incubated for 24 hours. After 24 hours, the medium was discarded and the cultured cells were incubated with $1 \mu \mathrm{g} / \mathrm{ml}$ LPS derived from E. coli (serotype 0111:B4, MilliporeSigma) in the culture medium for 24 hours. After 24 hours, the supernatants were collected and tested for the presence of proinflammatory cytokines TNF- $\alpha$ and IL- 6 by ELISA (R\&D Systems).

Real-time quantitative PCR. To further characterize the activation state of the microglia, proinflammatory cytokine gene expression (IL-6, TNF- $\alpha$, TGF- $\beta$, and IL-1 $\beta$ ) was investigated using real-time PCR. Total RNA from microglia was extracted using a RNeasy Mini kit (Qiagen). $1 \mu \mathrm{g}$ total RNA was reverse transcribed using a First-Strand cDNA Synthesis kit (Fermentas Life Sciences). Relative quantification of gene expression was performed by real-time PCR using iQ SYBR-Green Supermix on the iCycler iQ thermal cycler (Bio-Rad) following the manufacturer's protocols. GAPDH (glyceraldehyde-3-phosphate dehydrogenase) was used to normalize the quantitative experiments based on prior reference-gene suitability testing. GAPDH synthesis was shown not to be significantly different between groups. The specific ratio between the gene of interest and the reference gene was calculated for each sample.

Primer sequences were as follows: mouse GAPDH sense, GGCCTTCCGTGTTCCTAC; antisense, TGTCATCATATCTGGCAGGTT; mouse IL-6 sense, CAAAGCCAGAGTCCTTCAGA; antisense, GCCACTCCTTCTGTGACTCC; mouse TGF- $\beta$ sense, CTTTTGACGTCACTGGAGTTG; antisense, CAGTGAGCGCTGAATCGAA; mouse TNF- $\alpha$ sense, GCCTCTTCTCATTCCTGCTT; antisense, AGGGTCTGGGCCATAGAACT; and mouse $I L-1 \beta$ sense, GGGCCTCAAAGGAAAGAATC; antisense, TCTTCTTTGGGTATTGCTTGG. Results are expressed as the ratio between gene expression in the $\mathrm{PE}$ offspring and control offspring. 
Immunohistochemical, histological, and morphometric studies. Kidneys, hearts, and aortas from every experimental group were harvested and frozen in O.C.T. compound (Sakura Finetek). Cryostatic sections were cut and incubated with protein block serum-free (Dako, Agilent Pathology solutions) prior to immunohistochemical analysis to prevent nonspecific background staining. Kidney cryostatic sections (5 $\mu \mathrm{m})$ were then incubated with primary antibodies directed to mouse fibrinogen/fibrin (Abcam, ab34269), tissue factor (Abcam, ab151748), and complement C3b/iC3b/C3d (Hycult Biotech, mAb 3/26) followed by incubation with specific secondary IgG antibodies conjugated with FITC (Abcam).

LV sections $(10 \mu \mathrm{m})$ cut through the long axis were stained with antibodies for collagen type I (Acris antibodies GmbH R1038X) and MMP-9 (Abcam, ab38898) followed by Texas red-and FTIC-labeled secondary antibody to detect cardiac remodeling/fibrosis. ProLong Gold mountant with DAPI (Thermo Fisher) was used to stain nuclei. Actin filaments in cardiomyocytes were stained with the Cytopainter F-actin staining kit (green fluorescence, Abcam).

Digital images of transverse sections $(10 \mu \mathrm{m})$ were used to quantify the area of cardiomyocytes. Cardiomyocytes were outlined manually and filled using the paint bucket tool in Photoshop (Adobe Photoshop CC 2017). Images were opened in ImageJ (NIH), and cross-sectional area was measured. To help eliminate cells sectioned tangentially, cardiomyocytes with an aspect ratio of $\geq 1.2$ were excluded. Areas of 50 myocytes from 5 randomly selected microscope fields from the LV posterior wall from each experimental group were averaged to represent the myocyte area.

Frozen sections of aortas $(10 \mu \mathrm{m})$ were stained for collagen I (R1038X, Acris $\mathrm{GmbH})$ followed by a FITC-conjugated secondary antibody.

Sections of formalin-fixed paraffin-embedded kidneys were cut at $3 \mu \mathrm{m}$ and stained with antibodies against collagen type I (R1038X, Acris GmbH) and ASMA (clone 1A-4, MilliporeSigma) followed by Texas red- and FITC-conjugated secondary antibodies, respectively. A 0.1\% solution of Sudan Black B (MilliporeSigma) in 70\% ethanol was used in both frozen and paraffin-embedded kidney tissue to block autofluorescence.

Immunohistochemical staining for the calcium-binding protein lba-1 (Abcam, ab 5076) was used to investigate microglia activation in P30 brain tissue sections $(10 \mu \mathrm{m})$. A FITC-labeled secondary antibody was used. Ten slides per experimental group were examined and photographed under a fluorescence microscope (Nikon 50i) attached to a DigiSight Color digital camera system (Nikon DSRi1). Stained tissue areas were quantified using ImageJ (NIH).

Statistics. All statistical analyses were performed using Prism 6.0 software (GraphPad Software Inc.). Due to the fact that more than 2 groups were to be compared, the ANOVA followed by a post-hoc Bonferroni test were used in order to avoid multiple-comparisons error. Data are presented as raw data and mean \pm SD. In all cases, $P$ values equal to or less than 0.05 were deemed statistically significant.

Study approval. All animal studies were performed in accordance with the 1986 UK Home Office Animal Procedures Act. Local ethics committee approval was obtained from the Animal Welfare Ethical Review Board committee at St Thomas' Hospital.

\section{Author contributions}

GG designed the study. NG, MS, MU, JP, and GG performed experiments. GG analyzed and interpreted data. GG wrote the manuscript. JEC performed the ultrasound imaging studies.

\section{Acknowledgments}

This study was partially funded by Tommy's Charity and TheirWorld. We thank P. Seed for assistance with statistical analysis.

Address correspondence to: Guillermina Girardi, Pregnancy Laboratory, Department of Women and Children's Health, The Rayne Institute, 4th Floor Lambeth Wing, St Thomas' Hospital, King's College London, London SE1 7EH, United Kingdom. Phone: 44.0.207.188.1101; Email: guillermina.girardi@kcl.ac.uk.

1. Xu J, Murphy SL, Kochanek KD, Bastian BA. Deaths: final data for 2013. Natl Vital Stat Rep. 2016;64(2):1-119.

2. Kuklina EV, Ayala C, Callaghan WM. Hypertensive disorders and severe obstetric morbidity in the United States. Obstet Gynecol. 2009;113(6):1299-1306

3. McDonald SD, Malinowski A, Zhou Q, Yusuf S, Devereaux PJ. Cardiovascular sequelae of preeclampsia/eclampsia: a system- 
atic review and meta-analyses. Am Heart J. 2008;156(5):918-930.

4. Bellamy L, Casas JP, Hingorani AD, Williams DJ. Pre-eclampsia and risk of cardiovascular disease and cancer in later life: systematic review and meta-analysis. BMJ. 2007;335(7627):974.

5. Vikse BE, Irgens LM, Leivestad T, Skjaerven R, Iversen BM. Preeclampsia and the risk of end-stage renal disease. $N E n g l J$ Med. 2008;359(8):800-809.

6. Ray JG, Vermeulen MJ, Schull MJ, Redelmeier DA. Cardiovascular health after maternal placental syndromes (CHAMPS): population-based retrospective cohort study. Lancet. 2005;366(9499):1797-1803.

7. Mosca L, et al. Effectiveness-based guidelines for the prevention of cardiovascular disease in women--2011 update: a guideline from the American heart association. Circulation. 2011;123(11):1243-1262.

8. Singh J, Ahmed A, Girardi G. Role of complement component C1q in the onset of preeclampsia in mice. Hypertension. 2011;58(4):716-724.

9. Davis EF, et al. Cardiovascular risk factors in children and young adults born to preeclamptic pregnancies: a systematic review. Pediatrics. 2012;129(6):e1552-e1561.

10. Costantine MM, et al. Using pravastatin to improve the vascular reactivity in a mouse model of soluble fms-like tyrosine kinase1-induced preeclampsia. Obstet Gynecol. 2010;116(1):114-120.

11. Kumasawa K, et al. Pravastatin induces placental growth factor (PGF) and ameliorates preeclampsia in a mouse model. Proc Natl Acad Sci USA. 2011;108(4):1451-1455.

12. Costantine MM, et al. Safety and pharmacokinetics of pravastatin used for the prevention of preeclampsia in high-risk pregnant women: a pilot randomized controlled trial. Am J Obstet Gynecol. 2016;214(6):720.e1-720.e17.

13. Lefkou E, et al. Clinical improvement and successful pregnancy in a preeclamptic patient with antiphospholipid syndrome treated with pravastatin. Hypertension. 2014;63(5):e118-e119.

14. Brownfoot FC, et al. Effects of pravastatin on human placenta, endothelium, and women with severe preeclampsia. Hypertension. 2015;66(3):687-697.

15. Lefkou E, Mamopoulos A, Dagklis T, Vosnakis C, Rousso D, Girardi G. Pravastatin improves pregnancy outcomes in obstetric antiphospholipid syndrome refractory to antithrombotic therapy. J Clin Invest. 2016;126(8):2933-2940.

16. Furchgott RF, Zawadzki JV. The obligatory role of endothelial cells in the relaxation of arterial smooth muscle by acetylcholine Nature. 1980;288(5789):373-376.

17. Kirilov G, Zacharieva S, Alexandrov AS, Lozanov V, Mitev V. Increased plasma endothelin level as an endothelial marker of cardiovascular risk in patients with active acromegaly: a comparison with plasma homocysteine. Methods Find Exp Clin Pharmacol. 2009;31(7):457-461.

18. Penning $\mathrm{M}$, et al. Classical complement pathway activation in the kidneys of women with preeclampsia. Hypertension. 2015;66(1):117-125.

19. Sharma AK, Mauer SM, Kim Y, Michael AF. Interstitial fibrosis in obstructive nephropathy. Kidney Int. 1993;44(4):774-788

20. Kimura K, et al. Hypertensive glomerular damage as revealed by the expression of alpha-smooth muscle actin and non-muscle myosin. Kidney Int Suppl. 1996;55:S169-S172.

21. Redman CW, Sacks GP, Sargent IL. Preeclampsia: an excessive maternal inflammatory response to pregnancy. Am J Obstet Gynecol. 1999;180(2 Pt 1):499-506.

22. De Craemer D, Lobe E, Pauwels M, Verbeelen D, Van den Branden C. Angiotensin II administration causes enhanced expression of glomerulosclerosis-related markers and decreased renal antioxidant enzyme activities in rats. Exp Nephrol. 2001;9(2):125-132.

23. Schannwell CM, Zimmermann T, Schneppenheim M, Plehn G, Marx R, Strauer BE. Left ventricular hypertrophy and diastolic dysfunction in healthy pregnant women. Cardiology. 2002;97(2):73-78.

24. Cong J, Fan T, Yang X, Shen J, Cheng G, Zhang Z. Maternal cardiac remodeling and dysfunction in preeclampsia: a three-dimensional speckle-tracking echocardiography study. Int J Cardiovasc Imaging. 2015;31(7):1361-1368.

25. Martin FL, et al. Experimental mild renal insufficiency mediates early cardiac apoptosis, fibrosis, and diastolic dysfunction: a kidney-heart connection. Am J Physiol Regul Integr Comp Physiol. 2012;302(2):R292-R299.

26. Susic D, Nuñez E, Frohlich ED, Prakash O. Angiotensin II increases left ventricular mass without affecting myosin isoform mRNAs. Hypertension. 1996;28(2):265-268.

27. Fernández-Ruiz I. Immune system and cardiovascular disease. Nat Rev Cardiol. 2016;13(9):503.

28. McMaster WG, Kirabo A, Madhur MS, Harrison DG. Inflammation, immunity, and hypertensive end-organ damage. Circ Res 2015;116(6):1022-1033.

29. Fielding CA, et al. Interleukin-6 signaling drives fibrosis in unresolved inflammation. Immunity. 2014;40(1):40-50

30. Choudhry $\mathrm{N}$, et al. The complement factor $5 \mathrm{a}$ receptor 1 has a pathogenic role in chronic inflammation and renal fibrosis in a murine model of chronic pyelonephritis. Kidney Int. 2016;90(3):540-554.

31. Zhang C, Li Y, Wang C, Wu Y, Du J. Antagonist of C5aR prevents cardiac remodeling in angiotensin II-induced hypertension. Am J Hypertens. 2014;27(6):857-864.

32. Allison MA, et al. Relation of leptin to left ventricular hypertrophy (from the multi-ethnic study of atherosclerosis). Am J Cardiol. 2013;112(5):726-730.

33. Lee KW, Lip GY. Insulin resistance and vascular remodelling, in relation to left ventricular mass, geometry and function: an answer to LIFE? J Hum Hypertens. 2003;17(5):299-304.

34. Barker DJ. In utero programming of chronic disease. Clin Sci. 1998;95(2):115-128.

35. Winklewski PJ, Radkowski M, Wszedybyl-Winklewska M, Demkow U. Brain inflammation and hypertension: the chicken or the egg? J Neuroinflammation. 2015;12:85.

36. Cohen E, Wong FY, Horne RS, Yiallourou SR. Intrauterine growth restriction: impact on cardiovascular development and function throughout infancy. Pediatr Res. 2016;79(6):821-830.

37. Walsh SW, Vaughan JE, Wang Y, Roberts LJ. Placental isoprostane is significantly increased in preeclampsia. FASEB J. 2000;14(10):1289-1296.

38. Hayashi KG, Hosoe M, Takahashi T. Placental expression and localization of endothelin-1 system and nitric oxide synthases 
during bovine pregnancy. Anim Reprod Sci. 2012;134(3-4):150-157.

39. Kameda T, et al. Production of interleukin-6 by normal human trophoblast. Placenta. 1990;11(3):205-213.

40. Bulla R, Bossi F, Tedesco F. The complement system at the embryo implantation site: friend or foe? Front Immunol. 2012;3:55.

41. Salomon C, Rice GE. Role of exosomes in placental homeostasis and pregnancy disorders. Prog Mol Biol Transl Sci. 2017;145:163-179.

42. Kohli S, et al. Maternal extracellular vesicles and platelets promote preeclampsia via inflammasome activation in trophoblasts Blood. 2016;128(17):2153-2164.

43. Thum T, Mayr M. Review focus on the role of microRNA in cardiovascular biology and disease. Cardiovasc Res. 2012;93(4):543-544

44. Bytautiene E, et al. Prepregnancy obesity and sFlt1-induced preeclampsia in mice: developmental programming model of metabolic syndrome. Am J Obstet Gynecol. 2011;204(5):398.e1-398.e8.

45. Heltemes A, et al. Chronic placental ischemia alters amniotic fluid milieu and results in impaired glucose tolerance, insulin resistance and hyperleptinemia in young rats. Exp Biol Med (Maywood). 2010;235(7):892-899.

46. McDonnold M, et al. The effect of prenatal pravastatin treatment on altered fetal programming of postnatal growth and metabolic function in a preeclampsia-like murine model. Am J Obstet Gynecol. 2014;210(6):542.e1-542.e7.

47. Staff AC, et al. Pregnancy and long-term maternal cardiovascular health: progress through harmonization of research cohorts and biobanks. Hypertension. 2016;67(2):251-260.

48. Staff AC, Johnsen GM, Dechend R, Redman CW. Preeclampsia and uteroplacental acute atherosis: immune and inflammatory factors. J Reprod Immunol. 2014;101-102:120-126.

49. Nanovskaya TN, Patrikeeva SL, Paul J, Costantine MM, Hankins GD, Ahmed MS. Transplacental transfer and distribution of pravastatin. Am J Obstet Gynecol. 2013;209(4):373.e1-373.e5.

50. Agostinis $\mathrm{C}$, et al. An alternative role of $\mathrm{C} 1 \mathrm{q}$ in cell migration and tissue remodeling: contribution to trophoblast invasion and placental development. J Immunol. 2010;185(7):4420-4429.

51. Agostinis C, et al. Complement component C1q as potential diagnostic but not predictive marker of preeclampsia. Am J Reprod Immunol. 2016;76(6):475-481.

52. Barnabei MS, Palpant NJ, Metzger JM. Influence of genetic background on ex vivo and in vivo cardiac function in several commonly used inbred mouse strains. Physiol Genomics. 2010;42A(2):103-113.

53. Dutta S, Sengupta P. Men and mice: Relating their ages. Life Sci. 2016;152:244-248.

54. Mulvany MJ, Halpern W. Mechanical properties of vascular smooth muscle cells in situ. Nature. 1976;260(5552):617-619.

55. Feng M, DiPetrillo K. Non-invasive blood pressure measurement in mice. Methods Mol Biol. 2009;573:45-55.

56. Feng M, Whitesall S, Zhang Y, Beibel M, D'Alecy L, DiPetrillo K. Validation of volume-pressure recording tail-cuff blood pressure measurements. Am J Hypertens. 2008;21(12):1288-1291.

57. Marshall SM. Screening for microalbuminuria: which measurement? Diabet Med. 1991;8(8):706-711.

58. Shen XZ, et al. Microglia participate in neurogenic regulation of hypertension. Hypertension. 2015;66(2):309-316.

59. Schang AL, et al. Failure of thyroid hormone treatment to prevent inflammation-induced white matter injury in the immature brain. Brain Behav Immun. 2014;37:95-102.

60. Stence N, Waite M, Dailey ME. Dynamics of microglial activation: a confocal time-lapse analysis in hippocampal slices. Glia 2001;33(3):256-266. 